\title{
Research on Symbiotic Design Facing User Experience
}

\author{
Lin Weishi \\ Guangdong University of Science and Technology, Dongguan, Guangdong, 523000
}

Keywords: user experience; product; symbiotic design

\begin{abstract}
This paper analyzes the connotation of user experience, and comprehensively the symbiotic design concept; it finally puts forward the symbiotic design strategy facing user experience. Taking user experience as a prerequisite for symbiotic design can ensure products meet customers' requirements and also can let users feel pleasure during use, so as to improve the value of products and ensure the harmony between the user and the product.
\end{abstract}

\section{Introduction}

In recent years, with the rapid development of society, people's living standards have been significantly improved, and higher requirements have been put forward for products. Designers are paying more and more attention to user experience, putting users in the first place, and making users have a strong interest in products, so as to attract more users to participate in them and gradually expand the range of products. Therefore, designers should pay more attention to symbiotic design, so that products and users have strong stickiness, and users can create resonance through products, so as to improve product satisfaction.

\section{Analysis on the Connotation of User Experience}

In the stage that user experiences the product, user is the main body of the whole experience process. During the period of experience, users can know about product features and usage effectively, and make their emotion obtain a certain experience. User experience is mainly based on the requirement of user [1], to ensure that the product is consistent with user requirements, which not only can effectively stimulate users and enhance their purchase desire for products, but also can attract users' attention and improve users' interest in the product. User experience is a subjective process, and users have different literacy, different life experiences, making the process of experience different. The higher cultural quality of the user is, the higher the requirement of experience is. According to Maslow's hierarchy of needs, physiological need is one's lowest need. When this need is met, people will seek a higher level of demand. If this theory is used in product design, user experience need can be divided into functional need, emotional need, etc. In order to reach the level of emotional need, users need to have certain emotions and experiences as the basis, so that users can make their psychology more pleasant through products, thus obtaining satisfaction. The product itself has no emotional factors, which is the designer's reasonable design, and designers makes it have certain vitality. Therefore, when designing products, designers should effectively grasp users' needs and closely connect them with users, which will not only facilitate users' acceptance of products, but also enable products to resonate with users in psychological aspect and enrich users' emotional experience.

\section{Concept of Symbiotic Design}

The concept, symbiosis, was first put forward by German mycologist De Berri. Symbiosis means two things are interdependent. If they are separated, it can bring great influence to the two sides. Symbiosis is a common phenomenon in life, and its main purpose is that the two kinds of different things exist mutually and independently, and also connect with each other, with mutual influence [2]. Product is not an isolated object but has a close relationship with users. When users participate 
in product creation and experience, they can not only give full play to the unique personality of the product, but also effectively carry the users' emotion, and strengthen the connection between products and users. If users are lost, the product will also lose its original meaning. Similarly, when there is no product as the support, users cannot place their emotion in an object, so users' emotion cannot be well expressed. From this perspective, the close connection between users and products is a kind of symbiotic relationship. Only by combining the two can the communication between people and things be realized, so as to express the emotion. On product design, designers should penetrate the symbiotic thought, to effectively coordinate the relationship between products and users, making it more harmonious. In this process, users can be involved in the design and use, making the product have the user own characteristics, to improve the emotional expression of product based on meeting users' functional needs, so as to achieve resonance. In design, designers should not only fully consider the materials and ensure the health of users, but also fully display user experience. Taking emotional need as the focus of the whole design, on the one hand, can make the product design quality significantly improved, and on the other hand, the product can be more consistent with users’ psychology.

\section{Symbiotic Design Strategy Facing User Experience}

Symbiotic design with user experience as a center is a complex process, covered by several aspects. In the process of design, designers should follow certain principles, make market research, understand users' psychological needs, so as to ensure the rationality of symbiotic design, improve the effect of design, which is explored from the following several aspects:

\subsection{Symbiotic design of human and product in design}

In the design phase, symbiotic design is mainly reflected in the process that users deeply participate in the product design. The current information technology is relatively developed, and there is more convenient for people to get access to information. It has become the inevitable trend for users to participate in product design phase. Users can use information technology to understand product information, have a clear design idea, and put forward their ideas, opinions, which can not only improve the product's uniqueness, but also attract the attention of users [3], thus improving the product sales. Product customization is a common form. Before users purchase the product, enterprises select the corresponding material and determine product size through the relevant platform; according to users' requirements for product design, it can not only enhance users' satisfaction of products, but also make the product more fit the needs of users, so as to improve the utilization rate of the product. To realize the rapid design, designers can take two ways to operate. Firstly, according to the existing product type, products are configured, with the application of standard module to the product design; on the one hand, it can make the design more efficient; on the other hand, it can ensure that the product corresponds to users, so as to achieve the purpose of rapid design. Secondly, if the module combination cannot meet users' requirements, designers can conduct product transformation design based on the existing product model, and effectively configure the product through the dynamic product model, thus improving the design efficiency [4]. Designers can also effectively improve the product structure and functions according to users' demands, so as to make it more satisfying to users' psychology. When conditions permit, users should actively participate in the design, so as to not only enable users to have a good experience and feel the fun brought by the design, but also provide users with a good atmosphere, so that users can realize the symbiosis with the product, so as to obtain emotional experience.

\subsection{Symbiotic design of human and product in production}

In the handicraft industry era, the economic level is relatively low, and people often need to make products for life, making products closely connected with life. With the social development, people have experience larger changes in the mode of production, making design and production separate, leading to the lack of user experience of product and reducing the product experience value, which not only affects the perception of users for the product, but also influences the 
product's practice and appreciation. At present, the people-oriented concept has been gradually deepened, and become a mainstream idea in the society. Product makers begin to attach importance to user experience, enabling users to participate in the whole product production, and effectively displaying users' personality thoughts. There are two main forms making users participate in product production.

First, users buy the required parts and make corresponding products. Influenced by market economy, some enterprises take obtaining more economic benefits as the premise condition to make the product homogeneity in the market relatively serious. In order to make the product more fit their own needs, many users do the products by themselves, which not only can make the product with strong personality, but also can make it reach the level of emotional need. Users need to buy the necessary parts first and assemble them according to the drawing. In this process, users can feel fun brought by handy operation, and also can effectively exercise practical ability [5]. Now, DIY has become the important symbol to show the unique personality. With Slime as example, users can buy Slime, and carries on the DIY, making it into their favorite characters or graphics. This not only allows users to experience, challenge themselves, but can make them enjoy the pleasure of production, making them achieve psychological satisfaction.

Second, users participate in the production of products and determine the shape to achieve the integration of hands and heart. Hands-on production can not only cultivate the creativity of users, but also enable users to harvest and improve the value of products. In the process of production, when users obtain the original materials, they will have a desire to do something by hands. They will reasonably construct the products according to their inner thoughts and assemble the products. When they see the complete product, they will get a sense of satisfaction, which cannot be replaced by the finished product. Although the production process is not perfect, users have integrated their own emotions and effectively play their imagination during the production, which is of positive significance to the training of users' image thinking, for example, the bread lamp below.

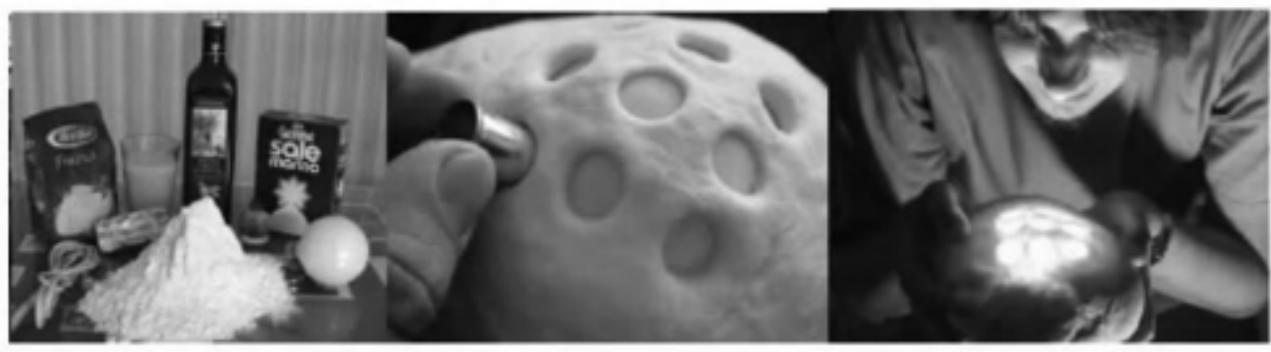

Fig.1

\subsection{Symbiotic design of human and product in use}

The characteristics of each user are different, and there will be differences in the use process. At this stage, symbiotic design can be divided into several forms: first, product reflects people's emotion. Product displays the user's emotion effectively through the user's skin, heart rate and other contents. At this stage, the user's emotion will be effectively expressed through facial expressions and gestures, and the product can receive the information through sensors, and then process it effectively, so as to be perceived by users. For example, a Japanese company has developed a product called "cat ear", which allows users to fully express their emotions when wearing it. For example, when users are sad, their ears will droop [6]; when users are happy, their ears will wave. Second, the product records users' usage habits. During the use, users' habits and interests can be recorded, which not only facilitates users' subsequent use, but also provides users with participation basis. Designers can provide effective feedback through this information and improve the product, so as to improve the product. 


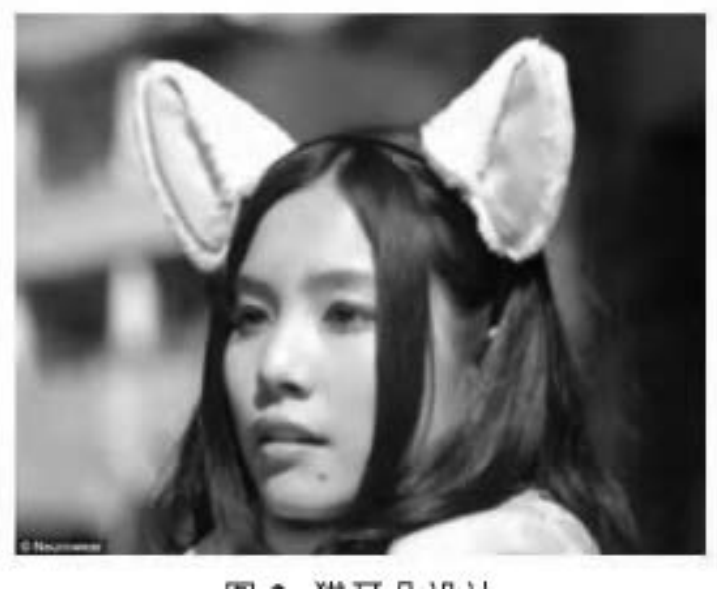

Fig. 2

\section{Conclusion}

Users participate in product production, and in use process, which can not only effectively show product features, but also make the product have stronger personal style; as a result, designers should realize the importance of symbiotic design, to take user as the main body, make them involved in the experience, make users produce strong intimacy for product, and then improve the value of product itself.

\section{References}

[1] Lan Cuiqin. Development of industrial design technology at intermediate level. Chinese National Academy of Arts, 2016.

[2] Dong Tiantian. Research on flat design in the context of contemporary thoughts. Southeast University, 2015.

[3] Wang Feng. Research on urban public art and its interactive design under the digital background. Jiangnan University, 2010.

[4] Yu Junping. Research on symbiotic design for user experience. Value Engineering,2 016,35(36):104-106.

[5] Gan Wei. Symbiotic interaction and automobile social design based on social media. Hunan University, 2015.

[6] Sen Wen. Research on design system and design practice based on cultural ecological view. Hunan University, 2017. 